\title{
Los medios de comunicación en el cambio político-social de finales del siglo XX
}

\section{Dinámica del mercado, transformación del Estado y proyecto de crecimiento nacional}

El nuevo modelo de crecimiento modernizador adoptado por el gobierno mexicano desde principios de la década de los años ochenta para salir de la crisis estructural de sus últimos ciclos de crecimiento, especialmente con la formación del Tratado de Libre Comercio entre México, Estados Unidos y Canadá ha ocasionado que la sociedad mexicana se adentre en un nuevo panorama histórico de inevitable globalización de la economía, de integración a modernas zonas hegemónicas y de radicales cambios comunitarios.

Dentro del escenario de los múltiples cambios profundos que ha vivido México en su nuevo modelo de desarrollo de finales de siglo XX, sobresale la importante transformación que se ha dado mediante el retiro creciente del Estado de la tarea estratégica de definir la dirección de la esfera pública y mediante la transferencia de esta dinámica rectora a la acción creciente de las leyes de la "mano invisible del mercado" para dirigir todos los ámbitos de nuestra vida nacional.

La rectoría de las leyes del mercado en el terreno comunicativo y cultural de nuestro país ha significado que entremos en la etapa de desarrollo nacional en la que se formula que ante la nueva globalidad internacional para ser eficientes, especialmente en el terreno comunicacional, hay que aceptar la propuesta de asimilar indiscriminadamente los principios del "laissez faire informativo" en el terreno comunicativo o cultural, o lo que es lo mismo, asimilar la mentalidad de que "lo que no deja dinero a nivel cultural, no sirve". Bases que, llevadas a sus últimas consecuencias, en la práctica real plantean el peligro de que en vez de fortalecer nuestro espíritu nacional frente a este periodo de apertura cultural,

1. Universidad Autónoma Metropolitana-Xochimilco, Departamento de Educación y Comunicación. 
dicho espíritu flexibilice y en ocasiones hasta erosione más sus valores para incorporarnos eficientemente como sociedad y sin restricción alguna a la nueva estructura de competencia y de acumulación de los mercados mundiales. ${ }^{2}$

Esto es, ante el florecimiento en nuestro país de las tesis modernizadoras que sostienen el adelgazamiento, la privatización, el repliegue, la desregulación, la globalización y la transnacionalización de todos los campos de lo público, hoy se formula, cada vez con mayor convencimiento, que la rectoría cultural de la sociedad mexicana no debe conducirse por la acción interventora de políticas del Estado, sino que debe ser conducida por el equilibrio "natural" y perfecto que produce el juego de las reglas del mercado libre entre productores y consumidores. De esta forma, para adecuar el espacio cultural de la sociedad mexicana a las nuevas necesidades del mercado, se altera la concepción tradicional de la actividad comunicativa que la comprendía como un producto social y se pasa, con mayor velocidad, a entenderla ahora como una simple mercancía más que debe estar regida por los principios de la oferta y la demanda.

Con las profundas transformaciones que ha introducido el proyecto de desarrollo de mercado se constata el tránsito radical de un proyecto de comunicación y culturas colectivas dirigidas por el Estado (por más deficiente, limitado y equivocado que haya sido), a una práctica informativa conducida por la lógica del mercado, que localiza su centro de acción en la generación de crecientes ganancias monetarias rápidas a costa de lo que sea.

\section{Reforma del Estado y transformación del sistema de comunicación: ¡no se le encuentra la cuadratura al círculo!}

\section{El fortalecimiento del proyecto de mercado como guía del crecimiento}

La sociedad mexicana a finales del milenio, no sólo ha requerido la aplicación de sólidas acciones económicas y políticas para superar la drástica crisis estructural de la nación, sino que sobre todo ha necesitado intentar realizar una reforma integral del Estado que permita que nuestro proyecto de crecimiento se reencauce por los senderos de la estabilidad perdida.

2. Para analizar algunos de los efectos que ha producido el impacto del modelo de mercado sobre las infraestructuras informativas de México en la etapa de modernización nacional, véase Ma. de la Luz Casas Pérez (1996, 1997), Roger Darío Sánchez Martínez, (1997), Carmen Gómez Mont, (1995) Delia Crovi Druetta (1995) Guillermo Bonfil Batalla, (1992), Carlos Monsivais, (1992), Mauricio de María y Campos, (1992), Eduardo Nivón, (1992), José Manuel Valenzuela Arce, (1992: 311-339). 
Urgente reforma del Estado que no sólo debería exigir la modificación de los aspectos legales del proceso electoral, de la impartición equilibrada de justicia, de la presencia intensiva de la sociedad en los espacios de representación pública, de la aplicación del nuevo federalismo, la autonomía de los poderes legislativo y judicial, el respeto al voto ciudadano, la limitación al poder presidencial, la negociación con los sectores insurrectos de Chiapas y Guerrero, etc., sino sobre todo la transformación del funcionamiento de sus sistemas culturales y de medios de comunicación colectivos, especialmente cuando la mayoría de las estructuras de comunicación presentes, desde el punto de vista de la participación social, ya son obsoletas. ${ }^{3}$ Debemos considerar que en la actualidad no puede existir un gobierno moderno y avanzado, sin la transformación democrática e integral de sus instituciones de cultura y medios de comunicación colectivos, ya que es a través de ellos como se realiza cotidianamente una de las principales formas de dirección y participación colectiva en comunidad.

Para realizar la reforma del Estado mexicano en materia de comunicación después de muchos años de promesas, de discursos, de esperas, de demagogias, de aperturas y de mínimos cambios por parte de los diversos órganos de rectoría del país, finalmente casi al concluir el siglo $\mathrm{XX}$, a principios del periodo de la gestión política 1995-2000 el gobierno mexicano en turno organizó en diversos momentos varias consultas públicas y acuerdos políticos sobre comunicación y cultura, con el fin de abrir a la participación de toda la sociedad la discusión sobre la estratégica reforma del Estado en materia de comunicación social y enriquecer dicho proyecto de cambio político.

A lo largo de la celebración de todos los eventos públicos nacionales para la discusión y transformación del Estado mexicano en materia de cultura y comunicación social en el periodo 1994-2000, se presentaron más de 11,800 diagnósticos y propuestas de todos los sectores: de la sociedad civil, política, gubernamental, empresarial y estatal de la República mexicana, para la transformación del Estado sobre la situación de la información colectiva. Dichas propuestas oscilaron desde la instauración del derecho de réplica en los medios, la formación del Código de Ética Periodística, la creación de la figura del ombudsman de la comunicación colectiva, el aprovechamiento democrático de $12.5 \%$ del tiempo oficial en los medios, prohibir la existencia de monopolios comunicativos, suprimir los mecanismos directos y velados de censura, hasta la revisión del otorgamiento de las concesiones, la apertura de los espacios comunicativos para la participación de la sociedad civil, la creación de un consejo nacional de medios de comunicación, la modificación total de la actual normatividad en materia de comunicación social, la ampliación de la red de medios culturales, etcétera.

Sin embargo, no obstante todas estas participaciones que presentaron el sentir de la sociedad civil acerca de la transformación del proyecto de comunicación nacional, casi tres años y medio después de haberse efectuado dichos procesos democráticos, al terminar el periodo de sesiones de la LVI legislatura

3. Cfr. El Financiero, 7 y 9 de febrero de 1996; El Universal, 10 de febrero de 1996. 
en la Cámara de Diputados, el Poder Legislativo no rescató ninguna de las innumerables propuestas de la sociedad para la modificación del sistema nacional de comunicaciones. ${ }^{4}$

Con este comportamiento político se demostró nítidamente que el Estado mexicano de finales del siglo XX no contó con voluntad política histórica para transformar el actual sistema de comunicación de la sociedad mexicana. Esto es, en materia de comunicación el sistema político mexicano continúa sin compromiso social para encontrarle la "cuadratura al círculo de la comunicación". En este sentido, al concluir el milenio asistimos todavía a la presencia de un Estado feudal mexicano que, como en la Edad Media, vía el rígido control de los medios de comunicación, actualmente no permite la participación de los grandes grupos en los procesos masivos de construcción de la conciencia social.

\title{
¿Qué hacer?: la sociedad civil y la formación de una política de comunicación para la sobrevivencia social
}

\begin{abstract}
Ante la evasiva histórica del Estado mexicano para transformar los medios de comunicación es necesario que la sociedad civil organizada presione sistemáticamente para reactivar el proceso de transformación democrática del Estado mexicano a través de la búsqueda e implementación de nuevas políticas para lograr la participación de la sociedad a través de otros procesos de comunicación y contribuir, con ello, a resolver las agudas contradicciones culturales que limitan el crecimiento equilibrado de nuestra nación. ${ }^{5}$ Es dentro de este contexto de
\end{abstract}

4. Cfr. El Financiero, 8 de junio de 1996.

5. Algunas de las contradicciones que se reflejan entre el nivel de conciencia y el proyecto de desarrollo nacional se manifiestan en la oposición creciente que se establece entre formación de la conciencia nacional y la difusión de los contenidos de los medios de comunicación, particularmente electrónicos, en México. En este sentido, debido a la cultura paralela que han formado los medios electrónicos de difusión en México en las últimas décadas, se observa que los niños mayores de seis años conocen más la información televisiva que la transmitida en la escuela primaria u otros órganos culturales. Por ejemplo, en el terreno de la realidad nacional, $77 \%$ de los pequeños retienen más frases como "La chispa de la vida" o "Recuérdame" y sólo $49 \%$ conserva otras como " iViva la Independencia!", "La solución somos todos" o "El respeto al derecho ajeno es la paz". De igual manera, mientras que casi la totalidad de los niños (92\%) retiene la imagen del gansito Marinela, menos de dos terceras partes $(64 \%)$ identifican la columna de nuestra Independencia o al cura Hidalgo. 63\% de los niños asocia fácilmente el tema de la tarjeta de crédito Carnet y sólo $43 \%$ reconoce la frase "El respeto al derecho ajeno es la paz". En resumen, observamos que de cada diez personajes que los niños identifican, sólo tres son de la historia de México.

En el campo de la historia, $67 \%$ de los niños identifican los días y horarios en que se transmiten sus programas favoritos de televisión, mientras que sólo $19 \%$ enuncia las fechas en que ocurrieron los acontecimientos más significativos de la historia nacional. Los super héroes de la televisión como la Mujer Maravilla, son más conocidos por los pequeños $(98 \%)$ que los héroes de la Revolución mexicana (33\%). El Chapulín Colorado es más evocado por los infantes (96\%) que los Niños Héroes de Chapultepec $(82 \%)$. Supermán está más presente 
renovación y transición al nuevo milenio que la acción de los medios desempeña un papel central para construir otras políticas culturales acordes con los problemas que debemos resolver para sobrevivir como comunidad y especie.

Para ello, es fundamental sustituir, dentro del contexto de la modernidad contemporánea, la idea de mantener una "política mínima" o "ultra mínima" en el campo de la comunicación social de México a través de la adopción indiscriminada de la dinámica del "laissez faire informativo"; para ahora introducir la concepción estratégica de establecer una "nueva política de comunicación básica para la sobrevivencia social", sin la cual no podrá construirse un orden civilizatorio superior que mantenga las condiciones elementales de la convivencia en comunidad, especialmente, en la medida en que en el país somos crecientemente sociedades de masas cada vez mayores. "Nueva política de comunicación básica para la sobrevivencia social" que no se limite a ejercer meramente la labor informativa espectacular, de diversión, de elevación del rating, de entretenimiento, etc., que ha promovido intensamente la dinámica de mercado; sino sobre todo que haga posible la participación democrática de la población en los procesos de creación de cultura y comunicación colectivos vía los complejos audiovisuales. Es decir, medios de comunicación al servicio del hombre y no el hombre al servicio del mercado vía la acción de los canales de información.

En este sentido, ante el panorama de creciente desigualdad comunicativa y espiritual que se establece en nuestra República con la bárbara acción de las leyes del mercado sobre el terreno comunicativo, es necesario que la sociedad civil presione para que el Estado mexicano cree las condiciones de otro equilibrio cultural a través de la elaboración de una nueva política de "cultura y

en la mente de los pequeños $(97 \%)$ que don Benito Juárez.

En materia religiosa, no obstante que nuestra sociedad es acentuadamente católica, más de la mitad de los niños (56\%) conoce el día en que se transmitía "Hogar dulce hogar", mientras que sólo $86 \%$ recuerda el día en que se celebra la Navidad. Mientras $55 \%$ de los niños pueden decir qué día pasaba el programa "Mis huéspedes", sólo $32 \%$ sabe la fecha en que se celebra la fiesta de la Virgen de Guadalupe. Los pequeños identifican mejor el logotipo de Sabritas $(86 \%)$ que una hostia (46\%).

En el área cívica, $87 \%$ de los infantes conocen los días en que se transmiten los programas cómicos y sólo $13 \%$ saben la fecha en que toma posesión el presidente de la República. Sólo $8 \%$ conoce la fecha en que el primer mandatario rinde su informe anual, mientras que $61 \%$ sí puede decir el día y la hora en que aparecen las series fantásticas. $83 \%$ de los niños identifican el logotipo de los pastelitos rellenos y sólo $63 \%$ conocen el Calendario Azteca. Mientras que $81 \%$ de los pequeños evocan el logotipo de los productos Marinela, sólo $66 \%$ identifican el escudo nacional. Las tres cuartas partes $(77 \%)$ de los niños identifican la imagen de chicles Adams y menos de una quinta parte (17\%) reconocen el Monumento a la Revolución. Finalmente, el logotipo de los chocolates Carlos V es más reconocido (77\%) que el Monumento del Ángel o el monumento a la Independencia (40\%). Instituto Nacional del Consumidor, 1982

Incluso este panorama cultural está por agravarse aún más por la apertura de nuestra economía al comercio internacional, dado el ingreso de México al Acuerdo General sobre Aranceles y Comercio (GATT), la firma del Tratado Norteamericano de Libre Comercio con Estados Unidos y Canadá y el debilitamiento de la política educativo-cultural del Estado mexicano. 
comunicación social para la sobrevivencia nacional", que actualmente no existe y que se requiere urgentemente para coexistir. Para ello hay que considerar que así como en el campo económico el Estado reprivatizó la banca estatal y paralelamente impulsó la banca de desarrollo para apoyar a los sectores más desfavorecidos, o que así como vendió empresas públicas para destinar fondos a los proyectos de equilibrio social vía el Programa Nacional de Solidaridad; ${ }^{6}$ ahora es necesario que así como ha permitido crecientemente el juego de los principios del mercado en el área cultural, apoye la dinámica de la comunicación social en el terreno comunicativo, que surge desde las necesidades de expresión y participación ideológica más apremiantes de los grupos mayoritarios del país.

Esta transformación comunicativa es especialmente importante efectuarla porque debemos considerar que así como una cantidad de actividades estratégicas para el desarrollo de la sociedad mexicana no pueden dejarse en manòs del sector mercantil, a riesgo de que desaparezcan, como es el caso de la medicina preventiva, la generación del transporte urbano público, el impulso a la ciencia básica, la producción de infraestructura urbana, ${ }^{7}$ etc., así tampoco puede delegarse al mercado la construcción de una "cultura social para la sobrevivencia nacional". Ello debido a que aunque paradójicamente, a largo plazo la planificación cultural es la inversión económica más rentable que existe en México, a corto plazo no es lucrativa dentro de los criterios de "ganancia pecuniaria" y de corto plazo que establece la actual concepción neoliberal de la vida vía la política de la mano invisible del mercado.

Así, la formación de una cultura ecológica, de una cultura de conservación de las especies que componen las cadenas de reproducción de la vida, de una cultura de la defensa de los ancianos, de una cultura del cuidado del planeta Tierra, de una cultura de la convivencia civil, de una cultura de revaloración de lo nacional, de una cultura de la promoción de la vida, etc., que son niveles de cerebralidad mínimos que requerimos construir y conservar en México para sobrevivir en sociedades de masas cada vez mayores, tendrán que ser creadas por un nuevo modelo de comunicación de servicio público, ya que para el modelo de comunicación mercantil no es rentable efectuarla.

En este sentido es fundamental tener presente que para que la sociedad mexicana se desarrolle a través del "moderno Estado mexicano", especialmente en los tiempos del Tratado de Libre Comercio y de la globalización total, es necesario aplicar muchas acciones administrativas, legislativas, políticas, infraestructurales, organizativas, etc.; pero además de estas actividades a nivel material, es indispensable ejecutar intensos y muy sólidos proyectos culturales orgánicos que respalden las acciones de gobierno y desarrollo nacional. Para ello es central que el Estado mexicano abandone sus tradicionales desviaciones "aristocráticas", "folcloristas", "elitistas" y "culturalistas" que ha practicado durante varias

6. Cfr. El Financiero, 5 de marzo de 1992; Excélsior, 7, 13, 1421 y 22 de marzo de 1992; Unomásuno, 8, 10, 11, 13 y 17 de marzo de 1992.

7. Cfr. Excélsior, 13 de abril, 15 de julio y 26 de noviembre de 1992; El Financiero, 13, 23 y 24 de noviembre de 1992. 
décadas y que han entendido la acción cultural de forma restringida como la expresión de las sensibilidades más refinadas en todos los órdenes de las artes y de los conocimientos, y la replantee ahora en un sentido amplio como la producción de una nueva cultura orgánica que responda a las principales problemáticas cotidianas que la sociedad mexicana tiene que resolver para sobrevivir.

Una cultura orgánica que supere la simple acción de alfabetizar, incrementar el nivel cuantitativo de la matrícula escolar básica, ampliar la educación formal, atender eficientemente los trámites instructivos, etc., y que prioritariamente se oriente a propiciar el desarrollo del país a través de la creación de mayores niveles de conciencia sobre las trabas que nos impiden crecer como socicdad.

Esto quiere decir que una estrategia para que los proyectos de desarrollo nacional puedan echar verdaderas raíces económicas y políticas en la población y así alcanzar, a nivel elemental, las metas propuestas, consiste en que el Estado, a través de los medios de comunicación electrónicos y particularmente de la televisión, tiene que formar en la conciencia de los ciudadanos una nueva cultura global para gobernar en el terreno ambiental, productivo, organizativo, urbano, de explotación de recursos naturales, de empleo, de vivienda, de energéticos, de agua, de salud, de participación, de generación de alimentos, etcétera.

Esto significa que en el momento actual que vivimos, de transición global del país, el Estado debe orientarşe prioritariamente a la formación de una cultura global para la sobrevivencia nacional y no para los goces exquisitos de la elite artística de nuestra sociedad. Hay que tener muy presente que el único sentido que pueden tener el arte y la cultura es el de incrementar los niveles de humanización de los hombres, no el de hacerlos más ajenos de sí mismos. Tenemos que combatir firmemente la posición que, con el fin de salir más rápidamente de nuestro subdesarrollo mental, el Estado ha generado en diversas ocasiones: el proceso artificial de propiciar la cultura por la cultura o el arte por el arte eliminando su relación con nuestras grandes contradicciones sociales.

Es por ello que la sociedad civil debe presionar enérgicamente para que el Estado mexicano canalice fundamentalmente la mayoría de su energía financiera, material, artística y espiritual hacia la producción de una nueva cultura agrícola para la producción de alimentos, que disminuya las más de nueve mil toneladas de nutrientes que actualmente importamos para existir. Una nueva cultura natural que evite que se sigan destruyendo más de las 200 especies animales y vegetales que se han aniquilado en lo que va de este siglo en nuestra República. Una nueva cultura local que aminore la migración de las más de diez mil personas por día hacia las principales ciudades del país. Una nueva cultura forestal que disminuya la erosión de más de 500 mil hectáreas anuales que se destruyen en nuestra reserva territorial. Una nueva cultura acuífera que permita darle una valor racional al agua para aprovecharla civilizadamente y ahorrar $30 \%$ del líquido potable que hoy se desperdicia irresponsablemente en las urbes. Una nueva cultura ecológica que contribuya a disminuir las más de seis mil toneladas diarias de partículas contaminantes que se acumulan en la atmósfera 
del Valle de México y las principales ciudades del país, y que silenciosamente cada vez más nos suprimen la vida.

Una nueva cultura femenina que permita la amplia participación social de las mujeres mexicanas, que son $50 \%$ de la población nacional. Una nueva cultura energética que nos lleve a vivir más justamente la transición por la que atraviesa el país al pasar de la era del petróleo a otra fase de desarrollo motriz. Una nueva cultura nutricional que posibilite disminuir la inanición de más de $60 \%$ de la población nacional, especialmente infantil, que permanentemente vive sin los mínimos alimenticios requeridos para sobrevivir. Una nueva cultura de la organización que integre alrededor de nuestros conflictos sociales básicos a los 85 millones de habitantes que vivimos en el país, pues todavía somos una sociedad que está por organizarse plenamente. Una nueva cultura que permita valorar y amar profundamente lo mexicano para afirmar nuestra cada vez más débil identidad nacional y evitar la rapidísima pérdida de nuestro proyecto cultural del país ante la apertura de fronteras. Una nueva cultura para las emergencias nacionales, especialmente de naturaleza sísmica en el Valle de México, cuando sabemos que los especialistas sismólogos de la Universidad Nacional Autónoma de México han anticipado la presencia de fuertes movimientos de las capas telúricas para los próximos años, como se ha comprobado una vez más con el temblor de tres grados en la escala de Richter del 25 de abril de 1989, y muchos más que los han precedido, etcétera. ${ }^{8}$

De aquí la necesidad urgente de reflexionar desde la sociedad civil sobre nuestra cultura y proponer la acción que deben ejercer los medios de comunicación dentro del proyecto de acelerada transformación del Estado mexicano, pues el vertiginoso proceso de la globalización mundial nos lleva a la creación de un nuevo orden cultural que está modificando los contenidos y las fronteras ideológicas de los actuales Estados nacionales.

En otros términos, hoy estamos obligados a preguntarnos con todo rigor desde la sociedad civil ihasta dónde a mediano y largo plazos el modelo de comunicación de mercado creará una cultura que propicie el verdadero crecimiento de nuestra sociedad o producirá un retroceso del avance del hombre? ¿qué acciones culturales debemos realizar para reforzar nuestra identidad nacional dentro de los marcos del proyecto de comunicación de mercado? icómo producir una cultura del desarrollo social dentro de la dinámica de la oferta y la demanda comunicativa? ¿cómo conciliar las presiones de un modelo comunicativo que deforma la estructura cultural para incrementar sus niveles de acumulación material, con la necesidad urgente de formar una cultura global para la sobrevivencia humana y el respeto a la vida? ¿cuáles deben ser los contenidos comunicativos de una política nacional de comunicación social en el México de final del siglo $\mathrm{XX}$ ?

8. Datos presentados en el programa radiofónico de Tomás Mojarro, "Palabras sin reposo", Radio UNAM, 25 de abril de 1989. 
De no efectuarse una profunda reforma democrática del Estado en materia de comunicación colectiva, el alma cultural de nuestra sociedad correrá el gran riesgo de quedar sepultada por los nuevos espejismos seductores que introduce el proceso de modernidad y sus derivados simbólicos parasitarios de esta nueva fase del desarrollo de la sociedad capitalista internacional. Con ello, los grandes límites naturales que por sí misma fija la economía de mercado sobre las dinámicas de comunicación televisiva, generarán más contradicciones culturales que las pretende resolver por la acción de la oferta y la demanda, y que sólo podrán ser resueltas con la introducción de la "racionalidad de la comunicación para la sobrevivencia social" en el campo de lo público.

En este sentido, si no se crea esta política de amplia participación social en el área comunicativa y cultural, cada vez más la sociedad mexicana vivirá el profundo divorcio entre necesidades materiales y espirituales de crecimiento social y la formación de la cerebralidad colectiva para resolverlas. Con esto, encontraremos que en plena fase de modernización nacional se habrán modificado las estructuras económicas, políticas, jurídicas, tecnológicas, etc., de nuestra sociedad; pero no se habrán transformado las estructuras mentales profundas que, en última instancia, son las que sostienen y le dan vida a nuestra comunidad nacional.

Bajo estas circunstancias la sociedad mexicana estará avanzando con los ojos vendados por un precipicio muy peligroso y dentro de algunos años veremos y sufriremos las consecuencias devastadoras que habrá dejado sobre nuestra conciencia y comportamientos colectivos la presencia de la lógica de mercado en el terreno cultural y espiritual de nuestro país.

\section{Bibliografía}

Bonfil Batalla, Guillermo (1992), "Dimensiones culturales del Tratado de Libre Comercio", en Gilberto Guevara Niebla y Nestor García Canclini (coords.) La educación y la cultura ante el Tratado de Libre Comercio, Nexos, Nueva Imagen, México, D.F.

Casas Pérez, Ma. de la Luz (1997), "El marco jurídico y la política de comunicación social en el marco del TLC", Anuario de Investigación de la Comunicación CONEICC IV, Consejo Nacional Para la Enseñanza e Investigación de las Ciencias de la Comunicación (CONEICC) y Universidad de Guadalajara, México, D.F.

(1996) "Identidad cultural y medios de comunicación: una cuestión no resuelta para Canadá y para México", Comunicación y Sociedad núm. 27, Departamento de Estudios de la Comunicación Social, Universidad de Guadalajara, mayo-agosto.

“Clinton, intervencionista astuto si concilia Estado y mercado", Excélsior, 26 de noviembre de 1992. 
“Clinton: ¿Un nuevo liberalismo social?", El Financiero, 24 de noviembre de 1992.

“Corrige J. Major a M. Thatcher y fortalece al Estado benefactor”, Excélsior, 15 de julio de 1992 .

Crovi Druetta, Delia, (coord.) (1995), Desarrollo de las industrias culturales en México y Canadá (Proyecto Monarca), Facultad de Ciencias Políticas y Sociales, Universidad Nacional Autónoma de México, México, D.F.

“De foros y más foros", El Universal, 10 de febrero de 1996.

De María y Campos, Mauricio (1992), "Las industrias culturales y del entretenimiento en el marco del Tratado de Libre Comercio", en La educación y la cultura ante el Tratado de Libre Comercio, Gilberto Guevara Niebla y Nestor García Canclini (coords.), Nexos Nueva Imagen, México, D.F.

"De nuevo sobre el liberalismo", Unomásuno, 10 de marzo de 1992.

"El liberalismo social fortalece la soberanía", Unomásuno, 13 de marzo de 1992.

“El liberalismo social se vive con el Pronasol”, Excélsior, 21 de marzo de 1992.

“El liberalismo social”, Excélsior, 7 de marzo de 1992.

"El liberalismo social: Nuestro camino", Unomásuno, 8 de marzo de 1992.

"El neointervencionismo estatal", El Financiero, 23 de noviembre de 1992.

"Entre la política y la economía. La condición de los serviles”, Excélsior, 13 de marzo de 1992.

"Es necesario crear una comisión que dé seguimiento a las propuestas", $E l$ Universal, 10 de febrero de 1996.

Gómez Mont, Carmen (1995). "La liberalización de las telecomunicaciones en México. De un sector estratégico al libre juego de las fuerzas del mercado", Anuario de Investigación de la Comunicación CONEICC II, Consejo Nacional Para la Enseñanza e Investigación de las Ciencias de la Comunicación y Universidad de Guadalajara, México, D.F.

"La comunidad cultural ante la reforma del Estado", El Financiero, 7 de febrero de 1996.

"La televisión y los niños. Conocimiento de la realidad televisiva vs. conocimiento de la realidad nacional", Cuadernos del Consumidor, Instituto Nacional del Consumidor, México, D.F., noviembre de 1982.

"Liberalismo social, nuestra filosofía; contra estatismo y neoliberalismo absorbente", El Financiero, 5 de marzo de 1992.

Monsiváis, Carlos (1992), "De la cultura mexicana en vísperas del Tratado de Libre Comercio", en La educación y la cultura ante el Tratado de Libre Comercio, Gilberto Guevara Niebla y Néstor García Canclini (coords.). Nexos Nueva Imagen, México, D.F.

“Ni la soberanía es obsoleta, ni existe un modelo único", Excélsior, 22 de marzo de 1992. 
Nivón Nivón, Eduardo (1992). "Propiedad intelectual y derechos de autor", en La educación y la cultura ante el Tratado de Libre Comercio, Gilberto Guevara Niebla y Néstor García Canclini (coords.). Nexos Nueva Imagen, México, D.F.

"Para los grandes medios de comunicación la noticia se genera desde el poder: Javier Elorriaga", El Financiero, 9 de febrero de 1996.

"Profundo rechazo en Wall Street a Clinton. Temor a un incremento de tasas de interés e inflación", El Financiero, 13 de noviembre de 1992.

"Propone el PRD crear un Consejo Nacional de Comunicación Social", El Financiero, 8 de junio de 1996.

“Qué del liberalismo", Unomásuno, 17 de marzo de 1992.

Radio UNAM, datos presentados en el programa radiofónico de Tomás Mojarro, "Palabras sin reposo", 25 de abril de 1989.

"Recursos a los marginados con mecanismos de mercado", Excélsior, 14 de marzo de 1992.

Sánchez Martínez, Roger Darío (1997), "Privatización de las telecomunicaciones: un estudio comparativo de las regulaciones en el sector de México y Chile”, Anuario de Investigación de la Comunicación CONEICC IV, Consejo Nacional Para la Enseñanza e Investigación de las Ciencias de la Comunicación y Universidad de Guadalajara, México, D.F.

"Sí al neoestatismo para guiar y no remplazar al mercado", Excélsior, 13 de abril de 1992.

Valenzuela Arce, José Manuel. "En la frontera norte de México: Tratado de Libre Comercio e identidad cultural", en La educación y la cultura ante el Tratado de Libre Comercio, Gilberto Guevara Niebla y Néstor García Canclini (coords.). Nexos Nueva Imagen, México, D.F., 1992.

“¿Neoliberalismo social?”, Unomásuno, 11 de marzo de 1992. 